
\title{
25 Research Soure \\ The Healthy Eating Plate advice for Migraine prevention: an interventional study
}

Claudia Altamura ( $\square$ c.altamura@gmail.com )

Universita Campus Bio-Medico di Roma https://orcid.org/0000-0002-5934-5535

\section{Gianluca Cecchi}

Universita Campus Bio-Medico di Roma

\section{Maria Bravo}

Universita Campus Bio-Medico di Roma

Alice Laudisio

Universita Campus Bio-Medico di Roma

\section{Paola Di Caprio}

Universita Campus Bio-Medico di Roma

Nicoletta Brunelli

Universita Campus Bio-Medico di Roma

\section{Giorgia Botti}

Universita Campus Bio-Medico di Roma

Matteo Paolucci

Universita Campus Bio-Medico di Roma

\section{Yeganeh Manon Khazrai}

Universita Campus Bio-Medico di Roma

\section{Fabrizio Vernieri}

Universita Campus Bio-Medico di Roma

\section{Research}

Keywords: migraine, diet, carb, healthy eating, metabolism

Posted Date: February 21st, 2020

DOI: https://doi.org/10.21203/rs.2.24183/v1

License: (a) (i) This work is licensed under a Creative Commons Attribution 4.0 International License. Read Full License 


\section{Abstract}

Background: migraine is a chronic neurological disorder with a high social impact. Several diets have been proposed to help managing migraine, with different outcomes. We aimed at evaluating the effect of education on the Healthy Eating Plate on migraine frequency and disability.

Methods: 240 consecutive people with migraine (18 - 72-year old, $84.5 \%$ female) were screened for participation in this interventional study. Migraine was diagnosed according to the International Classification of Headache Disorders. At three times of observation (screening $=\mathrm{T}-12,12$ weeks before the intervention; baseline $=\mathrm{T} 0$, time of educational intervention; and follow-up $=\mathrm{T} 12$, after 12 weeks from baseline) the enrolled people affected by migraine underwent anthropometric measurement, dietary patterns assessment, and migraine frequency and related disability evaluation (MIDAS, MIDAS A, MIDAS B). At T0 all enrolled people were educated about the Healthy Eating Plate by a nutritionist. The Healthy Eating Plate score was created to assess adherence to the dietary advice.

Results; 204 people with migraine were enrolled in the study, of these 119 people were still eligible at T0 while 97 people completed the evaluations at T12. From T0 to T12 we observed a reduction in body mass index and in monthly migraine days in the three months before the last evaluation (MIDAS A). People presenting a reduction of at least $30 \%$ in monthly migraine days were classified as responders. Responders significantly presented a reduction in red and processed meats and carb intake compared with non-responders. In addition, the Healthy Eating Plate scores were significantly higher in responders compared to non-responders, while no difference was observed for body mass index. The modification in carb consumption was also related to the variation in perceived disability (MIDAS score, $\rho=0.372$ with $p$ $<0.0001$ ) and in headache pain intensity (MIDAS B, $\rho=0.220, p=0.033$ ). Binary logistic regression confirmed the main effect of the Healthy Eating Plate score increase and total carb decrease on the responder state.

Conclusions This longitudinal study showed that adherence to the healthy eating plate advice, particularly the indication to reduce carb and red and processed meat consumption, is useful in migraine management, reducing monthly migraine days and disability.

Trial registration: ISRCTN, ISRCTN14092914. Registered 14 February 2020 - Retrospectively registered, http://www.isrctn.com/ISRCTN14092914

\section{Background}

Migraine is a neurological disorder with a high social impact on the general population because of its large diffusion and related disability.[1] Migraine pain is the epiphenomenon of a multifactorial cascade of mechanisms whose full understanding remains elusive. Diet has been often implied in its pathophysiology, as most people with migraine identify skipped meals or some particular foods as trigger factors. [2] Moreover, some dietary regimens based on certain food restrictions have shown promising results in migraine prevention [3] growing greatly the interest in the community of people with migraine.[4] 
At the same time, non-scientifically tested diets are diffusely proposed on the web. However, noncontrolled diets can have harmful implications, such as electrolyte or vitamin deficits and unbalanced nutritional intake. In this scenario, cross-sectional studies have recently shown that the adherence to healthy dietary habits is associated with lower frequency and duration of headaches. $[5,6]$

The Harvard T.H. Chan School of Public Health has proposed a renewed version of the food pyramid: the Healthy Eating Plate (HEP).[7] According to nutritional guidelines, the Healthy Eating Plate advice provides, in a simple format, detailed guidance to adopt correct eating patterns.

This study aimed at evaluating the effect of education on the Healthy eating Plate on monthly migraine days and disability. We hypothesized that the HEP advice can be of help in the management of migraine also as a strategy in add-on to pharmaceutical therapy.

\section{Methods}

\section{Study design}

We designed an unblinded longitudinal interventional study to evaluate the effect of HEP education in people with migraine.

\section{Participant selection}

Consecutive people with migraine treated at our Headache Centre were screened for enrolment from March 2018 to September 2019. Migraine was diagnosed according to the International Classification of Headache Disorders.[8] Screening visit included an accurate medical history interview, clinical examination and anthropometric assessment (height, weight, body mass index-BMI).

Among the 240 screened people affected by migraine, people were enrolled if the following inclusion and exclusion criteria were fulfilled:

- inclusion criteria: diagnosis of Migraine with Aura or Migraine without Aura, age> 18-year-old.

- exclusion criteria: BMI > 30, cancer, inflammatory bowel disease, celiac disease, type 1 diabetes, chronic renal insufficiency, and other neurological disorders.

We excluded 36 people with migraine for $\mathrm{BMI}>30$ that were referred to nutritional counseling.

For those people respecting screening criteria, additional criteria for exclusion were: failure to show at control visits and change in prophylactic therapy at baseline (dropouts).

Figure 1 summarizes the study design: a longitudinal study consisting of three visits: a screening visit ( $T$ 12), a baseline visit after 12 weeks (T0) and a follow-up visit (T12).

At T-12, the 204 eligible people signed the informed consent and filled a Frequency Food Questionnaire (FFQ) to assess their dietary habits and migraine disability clinical scales concerning the previous three 
months. All people received prophylactic treatment indications as appropriate.[9] At T0, 34 people failed to attend the control visit, the remaining eligible people underwent the assessments again (FFQ, migraine disability scales, BMI). They were all educated about the indications of the HEP by a nutritional biologist. Of these, 51 people requiring a change in preventive therapy were considered dropouts. At T12, 22 people did not show up. Finally, 97 people underwent all the evaluations and were included in the study. The study was approved by our Local Ethical committee (prot 6.18TS ComET CBM).

\section{Anthropometric measurements}

People' weight and height were measured at T-12, T0 and T12. People were weighed while wearing light clothes (i.e. no sweaters, jackets, or belts) and without shoes to avoid possible confounders for repeated measurement (including seasonal differences). Weight was measured to the nearest $0.1 \mathrm{~kg}$. Height was measured to the nearest $0.1 \mathrm{~cm}$ using a stadiometer, while the person was in a standing position with shoes removed, the shoulders were relaxed while looking straight ahead with the Frankfurt plane horizontal (scale and stadiometer, Fazzini, Milan, Italy).[10] BMI was calculated from the height and weight data, using the "weight $(\mathrm{kg}) /$ height $^{2}(\mathrm{~m})$ " equation.

\section{Migraine attack frequency and disability assessment}

Monthly migraine days and disability were assessed in all people at T-12, T0 and T12. Headache diaries were obtained from people recording the number of migraine days and of acute treatment drug consumption over the last month. All people were treated at our Headache center and already educated about corrected diary compilation. Migraine disability was assessed by a validated Italian version of the Migraine disability assessment score (MIDAS) questionnaire[11]. MIDAS is a semi-quantitative score measuring migraine days (MIDAS A), pain intensity (MIDAS B) and days of absence or reduced activity at work or on household due to headaches (MIDAS score) in the previous three months.

\section{Dietary Assessment and Education}

Food intake was assessed in all people at T-12, T0 and T12. Dietary intake over the previous 12 weeks was evaluated using a modified version of a semi-quantitative food frequency questionnaire (FFQ) validated in the Italian population. [12] This questionnaire includes a list of $110 \mathrm{food}$ items. For the aim of the current study, i.e. adherence to the healthy plate advice, we added in the "cereals and bread" section further items: spelt, barley and whole-wheat breakfast cereals with a total count of 113 food items. Before completing the FFQs, people were instructed on "serving size" amounts according to the Italian Society of Human Nutrition.[13] The FFQs were self-administered; however, a nutritionist was available in case participants felt insecure about compilation.

While filling the questionnaire people were asked to reply to some additional questions:

At $\mathrm{T}-12, \mathrm{~T} 0$, and $\mathrm{T} 12$ :

- Q1: "how many liters of water do you drink a day?" 
- Q2: "In your experience, do you think that a healthy diet can help improving headaches?

\section{At T12}

- Q3: "were you able to follow the healthy eating plate advice?"

- Q4: "were you able to reduce sugar daily consumption?"'

- Q5: "were you able to reduce salt use as flavor enhancer?"'

- Q6: "were you able to exercise at least 30 minutes a day"'

- Q7: "In your experience, do you think that the healthy eating plate advice helped you in improving headaches?"

People with migraine were required to answer "yes" or "no" to questions Q2-Q7.

At T0, after filling the questionnaires, all subjects were instructed by nutritional biologists about HEP and received a colored printed image of the HEP as well as the Italian written indications.[7]

To assess adherence to the HEP diet, we created a score (HEP score) ranging from 0 to 10 where one point was scored if each HEP indication was followed according to the results of the FFQ (Table 1).

Food intake frequency was calculated as weekly consumption, while water drinking on a daily basis. We calculated changes as differences in BMI, food intake frequency, HEP scores and disability measures (MIDAS score, MIDAS A and MIDAS B) from T0 to T12. Variation in the last month migraine days and drug intake frequencies was calculated as rates ((T12-T0)/T0). Since most people were already on pharmacological prophylactic treatment, we considered RESPONDERS people that achieved at least a $30 \%$ reduction in monthly migraine days from T0 to T12. The reduction in headache frequency is to be intended as a further reduction after the effect of the ongoing prophylactic treatment, unchanged throughout the study. This is in line with other migraine clinical settings where the expected benefit is mild.[14] Table 2 summarizes food groups. For the statistical analysis, we grouped as TOTAL CARBs the sum of refined cereals-potatoes and high carb breakfast - snacks intake frequencies, as TOTAL WHOLEGRAINs the sum of whole-grain breakfast and cereals intake frequencies, as HEALTHY PROTEINs the sum of fish and legumes intake frequencies, as OTHER PROTEIN the sum of white meat, egg, and cheese intake frequencies.

\section{Statistical Analysis}

Statistical analyses were performed using SPSS 25.0; SPSS Inc., Chicago, IL, USA. Differences were considered significant at the $p<.050$ level. The sample size was based on our previous experience with this design and further amplified.[15] Data distribution was assessed by the Kolmogorov-Smirnov test. Data of continuous variables are presented as mean values \pm standard deviation (SD). Median values with inter-quartile ranges (IQr) were provided for non-normally distributed variables. Analysis of variance 
(ANOVA) for normally distributed variables was performed according to RESPONDER status; otherwise, the nonparametric Mann-Whitney $U$ test was adopted. The two-tailed Fisher exact test was used for dichotomous variables. To assess changes over time in food intake, paired t-test or Friedman analysis of rank were adopted.

Multivariable logistic regression analysis was used to assess the association of RESPONDER status with age, sex, and all those variables which differed significantly $(P<.050)$ in comparison with NONRESPONDER.

\section{Results}

Table 3 summarizes demographic and anthropometric measures and migraine disability scales showing that the whole group presented a reduction in BMI and in migraine days in the three months before the last evaluation (MIDAS A). Prophylactic therapies were prescribed and continued until T12 in $74.6 \%$ of patients and distributed as follows: $22.2 \%$ tricyclic antidepressants, $14.1 \%$ beta-blockers, $10.1 \%$ calciumantagonists, $9.1 \%$ botulin toxin, $7.1 \%$ antiepileptic drugs.

Table 4 synthesizes food consumption along the three evaluation times: dietary habits differed only for a few food groups from T0 to T12. A positive response to Q2 was obtained in $62.9 \%$ of cases, in $53.6 \%$ to $\mathrm{Q} 3,77.3 \%$ to $\mathrm{Q} 4,73.2 \%$ to $\mathrm{Q} 5,50.5 \%$ to $\mathrm{Q} 6,60.8$ to $\mathrm{Q7}$.

Table 5 evidences anthropometric measures and food consumption frequencies in RESPONDERS compared with NON-RESPONDERS, highlighting that RESPONDERS significantly presented a reduction in red and processed meat and TOTAL CARB intake. In addition, the HEP scores were significantly higher in RESPONDERS $(-0.3$, IQr 1.57) compared to NON-RESPONDERS $(-1, \operatorname{IQ}$ r 1.92; $\mathrm{p}=.005)$, while no difference was observed for BMI. Spearman correlation showed that the variation in red and processed meat consumption related to monthly migraine days and drug intake change rates (respectively, $\rho=0.233$ with $p=0.024 ; \rho=0.233$ with $p=0.024)$. Similarly, the change in HEP score resulted related to the rate of monthly migraine day variation ( $\rho=-0.338$ with $p=0.001$, figure $2 A$ ). Finally, the variation in TOTAL CARBS consumption resulted related to monthly headache days and drug intake change rates (respectively, $\rho=$ 0.217 with $\mathrm{p}=0.033 ; \rho=0.220$ with $\mathrm{p}=0.033$ ) as well as to changes in perceived disability (MIDAS score, $\rho$ $=0.372, p<0.0001$-figure2B) and pain intensity (MIDAS B, $\rho=0.220, p=0.033$ ). To note, TOTAL CARBS intake and HEP score variations were not related to each other $(p=0.137)$.

TOTAL CARBS, red and processed meat consumption and HEP score changes were entered in a binary logistic regression model corrected for sex and age to assess their influence on RESPONDER state. The binary logistic regression confirmed the main effect of the HEP score increase and TOTAL CARBs decrease on the RESPONDER state (Table 6).

No interaction was observed between the RESPONDER state and the answers to Q2, Q5, Q6. A positive response to Q3 $(p=0.032), Q 4(p=0.037)$ and Q7 $(p=0.048)$ were more often observed in the RESPONDER group. 


\section{Discussion}

This study shows for the first time that education on a healthy diet might be beneficial in migraine prevention. In addition, the reduction in carbs and red and processed meat consumption seems to specifically play the main role. Different dietary regimens have been proposed so far as an adjunctive strategy in migraine management. Most of these diets are based on the avoidance of foods hypothesized to activate the attacks by influencing the plasma levels of molecules (such as CGRP, nitric oxide (NO), and serotonin) largely involved in migraine pathogenesis, or by affecting different aspects of brain homeostasis such as neuronal energy efficiency, excitability, and inflammation (also with an immunogenic response) or platelet aggregation.[16] Similarly, the ketogenic diet, which is able to exert a pharmacological action on cortical excitability by increasing the mitochondrial energy efficiency, activating GABAergic pathways and suppressing neuroinflammation, is based on the nearly complete elimination of carbs. $[17,18]$ The Healthy Eating Plate in line with nutritional guidelines does not prohibit any food; in contrast, it encourages varied nutrient consumption even though recommending a restriction for certain staples. A healthy diet seems to have beneficial effects also in other neurological disorders [19, 20]. The mechanism subtending these positive influences of a healthy diet on disease history can be numerous.

First, the Healthy Eating Plate diet can be considered at a low glycaemic index. The role of glucose metabolism in migraine physiopathology has been largely investigated, although a unique pathogenetic hypothesis is difficult to draw. [21] Cerebral metabolism cannot rely on glycogen storage, becoming in constant need of energetic supply from systemic circulation. A typical clinical example is fasting triggered headache. Hypoglycemia produces glucose shortage in the brain and catecholamines release resulting in sympathetic activation. Both mechanisms may favor migraine onset. As an apparent paradox, a low glycaemic index diet may be of benefit in migraine prevention.[22] Indeed, the reduction in blood glucose oscillation allows the stability of glucose regulating hormones that in turn have been involved in migraine pathogenesis. [21] A study on male rats observed that insulin and glucagon can alter the transmission of nociceptive inputs in the trigeminal-cervical complex suggesting another important potential neurobiological link between migraine and impaired metabolic homeostasis. [23] More recently, Kilic and colleagues reported that an inadequate brain glycogen can increases spreading depression susceptibility.[24] In line with these hypotheses, in our cohort, a net improvement in migraine attack frequency and related disability was strongly related to carb restriction (Fig. 2B). Moreover, responders reported having reduced added sugar more frequently than non-responders.

However, the reduction in carb intake is not the only aspect justifying the beneficial effect of the HEP diet. To note, the change in the HEP score did not relate to variation in carb consumption. One possible mechanism is the increase in nutrients with anti-inflammatory properties (fish, nuts, vegetables, and fruits) and the reduction in food with inflammatory potentials (red meat, dairies), together with adequate hydration proposed by the HEP diet. This more favorable balance together with a reduction in highly glycemic food can also have benefited the wealth of gut microbiota. The Gut-Brain Axis is the topic of an increasing number of studies. It has been implied in a variety of neurological and psychiatric disorders. A 
clear relation between gut microbiota and migraine is still to be established; however, its impact on the central and peripheral nervous systems supports a role in the migraine physiopathology.[25] Nevertheless, the HEP score did not increase significantly in the responder group, suggesting that the different items contributing to calculate the HEP score did not have the same impact on migraine (see Table 5).

Although only half of the people declared to have followed our nutritional advice, a positive reply was more frequently observed in responders. Interestingly, while we observed no relationship between an expected connection between migraine and diet at the beginning of the study (Q2), at the end of the study people in the responder's group affirmed more frequently that the HEP diet was of help for their migraine. The low adherence to the HEP advice and the relatively high number of dropouts were the only discouraging observations in this study. Beyond the self-reported adherence to the diet, the calculated HEP score was very low among all the evaluation times and paradoxically decreased from T0 to T12. Even if the HEP diet is mainly focused on the quality rather than the quantity of food, people may have encountered some difficulties in the everyday preparation of certain meals (especially vegetables). On the other side, at T12 people presented an overall reduction in BMI, an increase in whole-grain cereal consumption and in water intake and a reduction in red and processed meat, refined cereal product and sweetened beverage, suggesting that education on a healthy diet somehow increased their dietary awareness. To note, in our cohort weight loss was not associated with migraine improvement (Table 6). Similarly, salt reduction, which was self-reported in nearly $75 \%$ of cases, seemed not to exert any effect in our cohort.

\section{Conclusion}

Our study demonstrates that a comprehensive healthy diet can be beneficial in migraine management. General practitioners and headache specialists commonly recommend people to individuate food triggers and avoid their ingestion. Nevertheless, foods are specifically reported by people as triggers in a low percentage of cases.[26] Our study provides findings to motivate general practitioners and headache specialists to educate people with migraine also on a correct dietary style. This may have direct implications for migraine prevention and long term benefits for health.

\section{Abbreviations}

Healthy Eating Plate (HEP), body mass index (BMI), Frequency Food Questionnaire (FFQ), Migraine disability assessment score (MIDAS)

\section{Declarations}

Ethics approval and consent to participate: The study was approved by our Local Ethical committee (prot 6.18TS ComET CBM) and eligible people signed the informed consent.

Consent for publication: Not applicable 
Availability of data and materials: All data generated or analysed during this study are included in this published article (as additional file)

Competing interests: Authors have no conflict of interest

Funding: we did not receive any financial support

Authors' contributions: CA and YMK conceived the study. GC, MB, PDC and GB evaluated all the people with migraine and collected all clinical data. NB evaluated people with migraine. CA, AL and MP performed the statistical analysis. CA, YMK and FV drafted the manuscript.

Acknowledgements: None

\section{References}

1. GBD 2015 Neurological Disorders Collaborator Group VL, Abajobir AA, Abate KH, Abd-Allah F, Abdulle AM, Abera SF, et al. Global, regional, and national burden of neurological disorders during 1990-2015: a systematic analysis for the Global Burden of Disease Study 2015. The Lancet Neurology. 2017;16:877-97.

2. Martin VT, Vij B. Diet and Headache: Part 1. Headache. Blackwell Publishing Inc.; 2016;56:1543-52.

3. Di Lorenzo C, Coppola G, Sirianni G, Di Lorenzo G, Bracaglia M, Di Lenola D, et al. Migraine improvement during short lasting ketogenesis: a proof-of-concept study. European journal of neurology. 2015;22:170-7.

4. Slavin M, Ailani J. A Clinical Approach to Addressing Diet with Migraine Patients. Current Neurology and Neuroscience Reports. Current Medicine Group LLC 1; 2017.

5. Hajjarzadeh S, Mahdavi R, Shalilahmadi D, Nikniaz Z. The association of dietary patterns with migraine attack frequency in migrainous women. Nutritional Neuroscience. Taylor \& Francis; 2018;17.

6. Mirzababaei A, Khorsha F, Togha M, Yekaninejad MS, Okhovat AA, Mirzaei K. Associations between adherence to dietary approaches to stop hypertension (DASH) diet and migraine headache severity and duration among women. Nutritional neuroscience. 2018;1-8.

7. "The Nutrition Source" "the Harvard T.H. Chan School of Public Health." No Title [Internet]. Available from: https://www.hsph.harvard.edu/nutritionsource/healthy-eating-plate/

8. Headache Classification Committee of the International Headache Society (IHS) The International Classification of Headache Disorders, 3rd edition. Cephalalgia. 2018;38:1-211.

9. Silberstein SD, Holland S, Freitag F, Dodick DW, Argoff C, Ashman E, et al. Evidence-based guideline update: Pharmacologic treatment for episodic migraine prevention in adults: Table 1. Neurology. 2012;78:1337-45.

10. Gibson RS. Principles of Nutritional Assessment. Oxford University Press; 2005. 
11. D’Amico D, Grazzi L, Usai S, Raggi A, Leonardi M, Bussone G. Disability in chronic daily headache: state of the art and future directions. Neurological sciences: official journal of the Italian Neurological Society and of the Italian Society of Clinical Neurophysiology. 2011;32 Suppl 1:S71-6.

12. Marventano S, Mistretta A, Platania A, Galvano F, Grosso G. Reliability and relative validity of a food frequency questionnaire for Italian adults living in Sicily, Southern Italy. International journal of food sciences and nutrition. 2016;67:857-64.

13. SINU-Italian Society of Human Nutrition. No Title [Internet]. SICS Editore Srl. 2014. Available from: http://www.sinu.it/public/20141111_LARN_Porzioni.pdf

14. Vernieri F, Paolucci M, Altamura C, Pasqualetti P, Mastrangelo V, Pierangeli G, et al. Onabotulinumtoxin-A in Chronic Migraine: Should Timing and Definition of Non-Responder Status Be Revised? Suggestions From a Real-Life Italian Multicenter Experience. Headache. Blackwell Publishing Inc.; 2019;59:1300-9.

15. Altamura C, Botti G, Paolucci M, Brunelli N, Cecchi G, Khazrai M, et al. Promoting healthy eating can help preventing migraine: a real-life preliminary study. Neurological Sciences. 2018;

16. Razeghi Jahromi S, Ghorbani Z, Martelletti P, Lampl C, Togha M. Association of diet and headache. The Journal of Headache and Pain. 2019;20:106.

17. Di Lorenzo C, Pinto A, lenca R, Coppola G, Sirianni G, Di Lorenzo G, et al. A Randomized Double-Blind, Cross-Over Trial of very Low-Calorie Diet in Overweight Migraine Patients: A Possible Role for Ketones? Nutrients. 2019;11.

18. Augustin K, Khabbush A, Williams S, Eaton S, Orford M, Cross JH, et al. Mechanisms of action for the medium-chain triglyceride ketogenic diet in neurological and metabolic disorders. The Lancet Neurology. Elsevier; 2018;17:84-93.

19. Fitzgerald KC, Tyry T, Salter A, Cofield SS, Cutter G, Fox R, et al. Diet quality is associated with disability and symptom severity in multiple sclerosis. Neurology. 2017;90:e1-11.

20. Petersson SD, Philippou E. Mediterranean Diet, Cognitive Function, and Dementia: A Systematic Review of the Evidence. Advances in Nutrition. Oxford University Press (OUP); 2016;7:889-904.

21. Gross EC, Lisicki M, Fischer D, Sándor PS, Schoenen J. The metabolic face of migraine - from pathophysiology to treatment. Nature Reviews Neurology. Nature Publishing Group; 2019. p. 627-43.

22. Evcili G. Early and Long Period Follow-up Results of Low-Glycemic Index Diet for Migraine Prophylaxis. Ağrı - The Journal of The Turkish Society of Algology. 2018;30:8-11.

23. Martins-Oliveira M, Akerman S, Holland PR, Hoffmann JR, Tavares I, Goadsby PJ. Neuroendocrine signaling modulates specific neural networks relevant to migraine. Neurobiology of Disease. 2017;101:16-26.

24. Kilic K, Karatas H, Dönmez-Demir B, Eren-Kocak E, Gursoy-Ozdemir Y, Can A, et al. Inadequate brain glycogen or sleep increases spreading depression susceptibility. Annals of Neurology. John Wiley and Sons Inc.; 2018;83:61-73.

25. Slavin M, Li HA, Frankenfeld C, Cheskin LJ. What is Needed for Evidence-Based Dietary Recommendations for Migraine: A Call to Action for Nutrition and Microbiome Research. Headache. 


\section{Tables}

Table 1 . The healthy eating plate score

\begin{tabular}{|c|c|c|}
\hline Item & Yes & No \\
\hline Water per day $\geq 2 \mathrm{~L}$ & 1 & 0 \\
\hline At least 2 servings of seasonal fresh fruits per day & 1 & 0 \\
\hline At least 3 servings of vegetables per day & 1 & 0 \\
\hline No more than 2 servings of milk/dairy per day & 1 & 0 \\
\hline $\begin{array}{l}\text { At least } 8 \text { servings per week of whole grains in the mean AND a ratio whole grains/refined grain and } \\
\text { potatoes consumption }>1\end{array}$ & 1 & 0 \\
\hline Having for breakfast whole grains cereals or biscuits at least 5 times per week & 1 & 0 \\
\hline $\begin{array}{l}\text { Less than } 3 \text { servings a day of fat dressing AND a ratio healthy vegetable oils/partially hydrogenated } \\
\text { oils and animal fats }>3\end{array}$ & 1 & 0 \\
\hline Having no more than 1 sweetened beverage (including packaged fruit juice) per week & 1 & 0 \\
\hline Having 1-2 serving of nuts as a snack/day & 1 & 0 \\
\hline \multicolumn{3}{|l|}{ SUBTOTAL } \\
\hline \multicolumn{3}{|l|}{ Healthy protein sub-score: } \\
\hline Cheese no more than 1 serving per week & 1 & 0 \\
\hline 1 or 2 Eggs per week & 1 & 0 \\
\hline Fresh fish (including shellfish) at least 2 servings per week & 1 & 0 \\
\hline Beans at least 2 servings per week & 1 & 0 \\
\hline Poultry: 2 servings per week & 1 & 0 \\
\hline Red meat or processed meat once a week or less & 1 & 0 \\
\hline \multicolumn{3}{|l|}{ Protein subtotal } \\
\hline PROTEIN SCORE & \multicolumn{2}{|c|}{ Protein subtotal/6 } \\
\hline TOTAL & \multicolumn{2}{|c|}{$\begin{array}{l}\text { SUBTOTAL + PROTEIN } \\
\text { SCORE }\end{array}$} \\
\hline
\end{tabular}

Table 2 


\begin{tabular}{|c|c|}
\hline Food groups & Food Items \\
\hline Fresh fruits & All kind of fresh fruits \\
\hline Vegetables & All kind of vegetables (excluding potatoes) \\
\hline Milk and Diary & Milk, Yogurt, Mozzarella \\
\hline Whole grain cereals & Whole grain pasta, whole grain rice, spelt, barley, whole grain bread \\
\hline $\begin{array}{l}\text { Refined cereals- } \\
\text { potatoes }\end{array}$ & Refined grain Pasta, Refined grain Rice, Bread \\
\hline $\begin{array}{l}\text { High carb breakfast- } \\
\text { snacks }\end{array}$ & $\begin{array}{l}\text { Confectionary sweet products, croissants, refined grain biscuits, refined grain breakfast cereals, cakes, } \\
\text { French fries, fried rice balls }\end{array}$ \\
\hline $\begin{array}{l}\text { Whole grain } \\
\text { breakfast }\end{array}$ & Whole grain breakfast cereals, whole-grain biscuits \\
\hline Vegetable oils & Olive oil, Corn Oil, Seed Oil \\
\hline $\begin{array}{l}\text { Animal fat and } \\
\text { margarine }\end{array}$ & Butter, lard, margarine \\
\hline Cheese & Soft cheese and seasoned cheese (goat and cow milk) \\
\hline Egg & Egg \\
\hline Legumes & Different kind of beans, peas, lentil \\
\hline White meat & Chicken, turkey \\
\hline $\begin{array}{l}\text { Red and processed } \\
\text { meat }\end{array}$ & Beef, lamb, pork, sausage, ham, salami, and any processed meat \\
\hline Fish & Fresh or Frozen fish (or mussel) \\
\hline Sweetened beverage & Soft drinks, confectionary juices \\
\hline Nuts & walnuts, chestnuts, peanuts, hazelnuts, pistachio, almonds, seeds \\
\hline Alcohol & Wine, beer, spirits \\
\hline
\end{tabular}

Table 3. Demographic, anthropometric measures and migraine disability scales 


\begin{tabular}{|c|c|c|c|c|}
\hline $\mathrm{n}=97$ & $\mathrm{~T}-12$ & $\mathrm{~T} 0$ & $\mathrm{~T} 12$ & $\mathrm{p}$ \\
\hline Sex, F (\%) & 84.5 & - & - & \\
\hline Age, yrs (mean, SD) & $42.08(12.93)$ & - & - & \\
\hline BMI, kg/m² (median, IQr) & $23.2(5.30)$ & $23.21(5.65)$ & $23.14(5.18)$ & .009 \\
\hline Migraine days/ last month (median, IQr) & $7(8)$ & $7(7)$ & $6(7.5)$ & n.s. \\
\hline Drug intake/last month (median, IQr) & $6(8.5)$ & $6(8)$ & $6(9,5)$ & n.s. \\
\hline MIDAS - A (median, IQr) & $18(20)$ & $18(19)$ & $15(19.5)$ & .001 \\
\hline MIDAS - B (median, IQr) & $7(2)$ & $7(3)$ & $7(2)$ & n.s. \\
\hline MIDAS score (median, IQr) & $14(26)$ & $13(22)$ & $15(34.75)$ & n.s. \\
\hline
\end{tabular}

Table 4. The Healthy Eating Plate score and Food consumption frequency 


\begin{tabular}{|c|c|c|c|c|}
\hline $\mathrm{n}=97$ (median, IQr) & $\mathrm{T}-12$ & T0 & T12 & p \\
\hline Water intake, L/day & $1.00(0.50)$ & $1.00(0.5)$ & $2.00(1.00)$ & $<.0001$ \\
\hline Fresh fruits, serving/week & $7(10.5)$ & $7(10)$ & $7(8.5)$ & n.s. \\
\hline Vegetables, serving/week & $11(8)$ & $10(8)$ & $10(7)$ & n.s. \\
\hline Milk and diary, serving/week & $4(7.5)$ & $7(8)$ & $6(7)$ & n.s. \\
\hline Whole grain cereals, serving/week & $1(5.5)$ & $1(6)$ & $3.5(7)$ & .001 \\
\hline Refined cereals-potatoes, serving/week & $12(12)$ & $12(11.5)$ & $9(9)$ & $<.0001$ \\
\hline Whole grain breakfast, serving/week & $0(3.5)$ & $0(2)$ & $2(6.5)$ & .002 \\
\hline High carb breakfast - snacks, serving/week & $2(6)$ & $2(7)$ & $2(69$ & ns \\
\hline Vegetable oils, serving/week & $14(6.25)$ & $14(6)$ & $14(7)$ & ns \\
\hline Animal fat and margarine, serving/week & $0(1)$ & $0(1)$ & $0(0)$ & ns \\
\hline Cheese, serving/week & $4(4)$ & $4(5)$ & $4(6)$ & ns \\
\hline Eggs, serving/week & $1(1)$ & $1(1)$ & $1(1)$ & ns \\
\hline Legumes, serving/week & $1(1)$ & $1(1)$ & $1(1)$ & ns \\
\hline Fish, serving/week & $1(1)$ & $1(1)$ & $1(1)$ & ns \\
\hline White meat, serving/week & $2(3)$ & $2(3)$ & $2(3)$ & ns \\
\hline Red and processed meat, serving/week & $4(3)$ & $4(4)$ & $3(2)$ & $<.0001$ \\
\hline Sweetened beverage, serving/week & $1(4)$ & $1(2)$ & $0(2)$ & .006 \\
\hline Nuts/ serving/week & $1(4)$ & $1(4)$ & $1(4)$ & ns \\
\hline Alcohol/ serving/week & $0(2)$ & $0(2)$ & $0(2)$ & ns \\
\hline Healthy eating plate score & $3.5(1.33)$ & $4.67(2.05)$ & $4.33(1.84)$ & $<.0001$ \\
\hline
\end{tabular}

Table 5. Changes in food group weekly intake in responders compared with non-responders. 


\begin{tabular}{|l|c|c|c|}
\hline Food group (median, IQr) & Responders (n=33) & Non-responders (n=64) & $\mathrm{p}$ \\
\hline Water intake, L/day & $0(0.5)$ & $0.8(0,5)$ & $\mathrm{ns}$ \\
\hline Fresh fruits, serving/week & $0(5)$ & $0(3)$ & $\mathrm{ns}$ \\
\hline Vegetables, serving/week & $14(13)$ & $10(17)$ & $\mathrm{ns}$ \\
\hline Milk and diary, serving/week & $0(1)$ & $0(2)$ & $\mathrm{ns}$ \\
\hline TOTAL WHOLE-GRAINs, serving/week & $6(8)$ & $1.5(5.75)$ & $\mathrm{ns}$ \\
\hline TOTAL CARBs, serving/week & $-4.5(10)$ & $-3(12.75)$ & .015 \\
\hline Vegetable oils, serving/week & $0(10.25)$ & $0(0.75)$ & $\mathrm{ns}$ \\
\hline Animal fat and margarine, serving/week & $0(1)$ & $0(0)$ & $\mathrm{ns}$ \\
\hline Red and processed meat, serving/week & $-1.5(3)$ & $-0.5(3)$ & $\mathrm{ns}$ \\
\hline HEALTHY PROTEINs, serving/week & $-0.5(7)$ & $0(3)$ & $\mathrm{ns}$ \\
\hline OTHER PROTEINs, serving/week & $1(6.75)$ & $0(1.75)$ & $\mathrm{ns}$ \\
\hline Sweetened beverage, serving/week & $-0.5(1.25)$ & $(1.25)$ & \\
\hline Nuts, serving/week & $0(2)$ & & \\
\hline Alcohol, serving/week & & & \\
\hline
\end{tabular}

Table 6. Binary logistic regression of RESPONDER state on age, sex, TOTAL CARBs intake, Red and processed meat intake and healthy plates score T0-T12 variations

\section{Figures}




\begin{tabular}{|l|c|c|c|c|c|c|c|c|}
\hline & & & & & & & \multicolumn{2}{|c|}{$95 \%$ C.I. } \\
\cline { 7 - 10 } & B & S.E. & Wald & gl & Sign. & Exp(B) & Lower limit & Upper limit \\
\hline Age & .011 & .020 & .330 & 1 & .566 & 1.012 & .973 & 1.052 \\
\hline Sex & -.288 & .756 & .145 & 1 & .704 & .750 & .170 & 3.301 \\
\hline TOTAL CARBs & -.071 & .033 & 4.641 & 1 & .031 & .931 & .873 & .994 \\
\hline Red and processed meat & -.110 & .085 & 1.668 & 1 & .197 & .896 & .758 & 1.059 \\
\hline Healthy eating plate score & .542 & .225 & 5.788 & 1 & .016 & 1.720 & 1.106 & 2.675 \\
\hline Costant & 1.230 & .921 & 1.784 & 1 & .182 & .292 & & \\
\hline
\end{tabular}

T-12

BMI

FFQ

T-12 disability scales preventive therapy
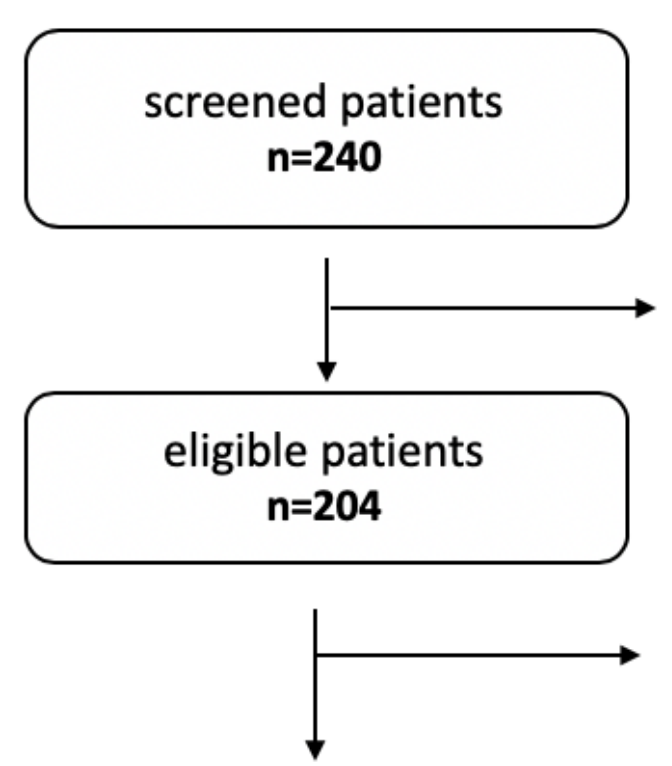

FFQ BMI

TO disability scales healthy plate advices

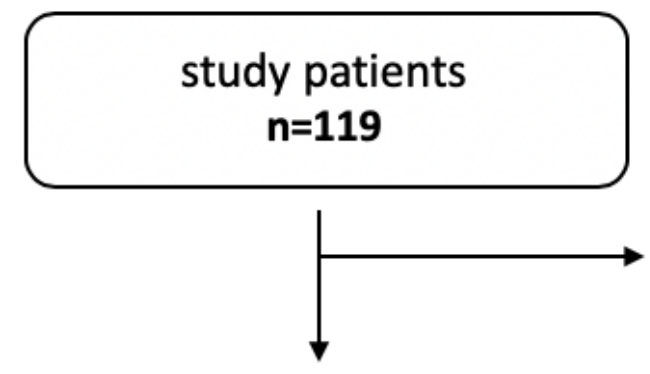

T12

FFQ BMI disability scales final data set $\mathrm{n}=\mathbf{9 7}$ ineligible patients

$B M \mid>30 n=12$

Other exclusion criteria $n=24$

\section{drop-out}

change in preventive therapy $n=51$ failed to show $n=34$

\section{drop-out}

failed to show $n=22$

\section{Figure 1}



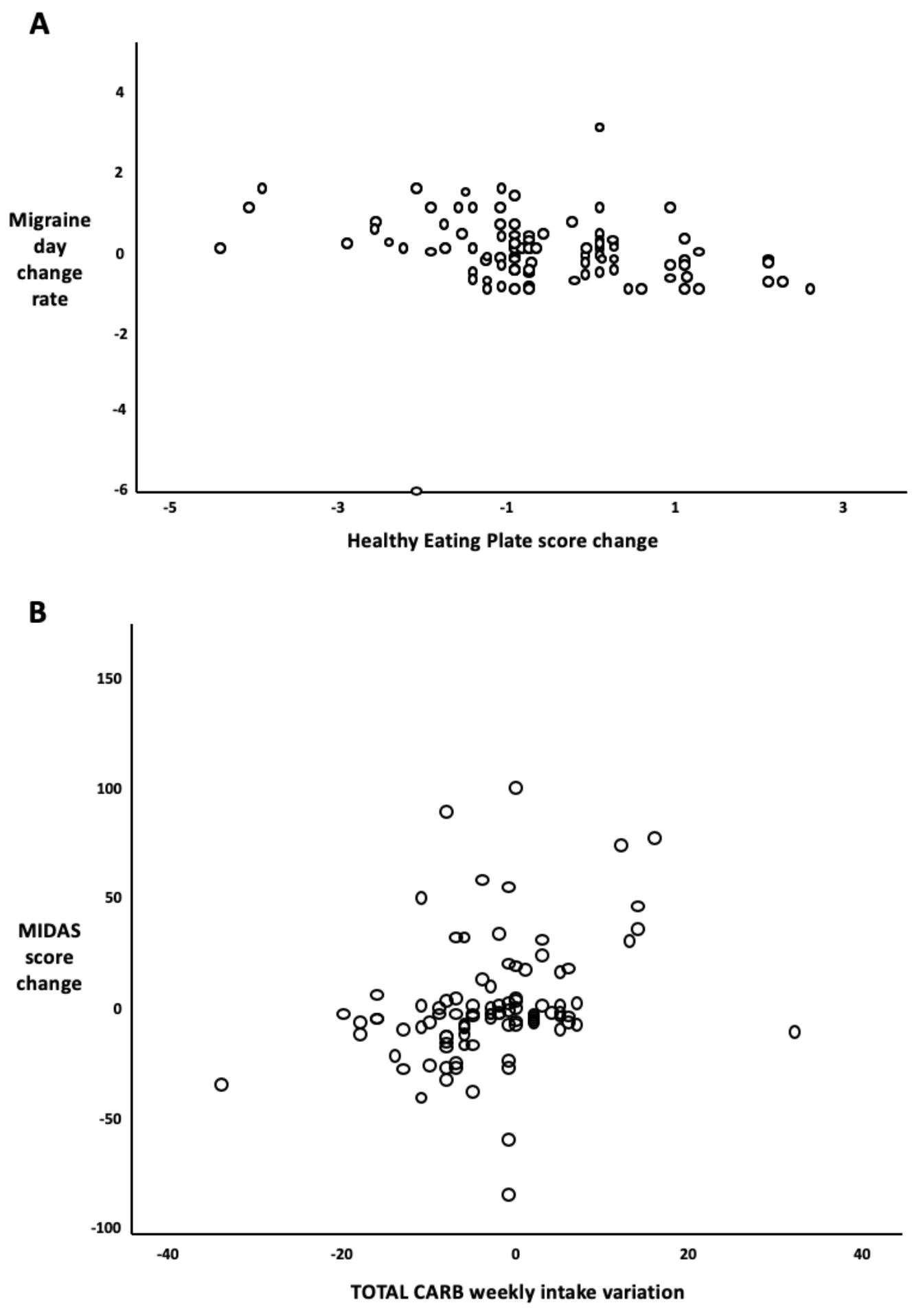

Figure 2

Scatterplot showing the relationship between HEP score variation and the rate of change in migraine days (A) and between changes in TOTAL CARB consumption and in MIDAS score (B) from T0 to T12.

\section{Supplementary Files}


This is a list of supplementary files associated with this preprint. Click to download.

- STROBECHECKLIST.doc

- DATASETHEALTHYEATING.xIsx 